\title{
KCTD15 Gene Expression Regulates Adipocyte Differentiation and Is Associated with Obesity in Humans
}

Jing Xu

Army Medical University

Xu Zhao

Beijing Institute of Genomics

Ying Yang

Army Medical University

Kebin Zhang

Army Medical University

Min Wang

Army Medical University

Zihui Xu ( $\boldsymbol{D}$ zihuixu@yeah.net)

Army Medical University https://orcid.org/0000-0001-8251-4774

Research article

Keywords: White adipose tissue; KCTD15; adipogenesis; Obesity

Posted Date: May 1st, 2020

DOI: https://doi.org/10.21203/rs.2.24498/v2

License: (c) (1) This work is licensed under a Creative Commons Attribution 4.0 International License.

Read Full License 


\section{Abstract}

Background: It has been substantiated by Genome-wide association studies (GWAS) that genetic disparity influences the fat levels and obesity risk in various populations.

Objective: A meta-analysis of 15 genome-wide association studies identified regularly-used obesityrelated variables at eight gene loci, inclusive of FTO, MC4R, TMEM18, KCTD15, GNPDA2, SH2B1, MTCH2 and NEGR1. However, the expression profiles of eight obesity-related genes remain largely unknown.

Methods: Based on the fundamental propositions specified in the Declaration of Helsinki, adipose tissue specimens were collected from 23 obese and 16 normal weight subjects, and the expression profiles of eight obesity-related genes identified by GWAS were examined in these white adipose tissues (WAT). The effects of KCTD15 gene on adipocyte differentiation in mature 3T3-L1 adipocytes were assessed.

Results: Among the obesity-related genes identified by GWAS, SH2B1, FTO, TMEM18 and KCTD15 were expressed at lower level in WAT of obese subjects compared to normal weight subjects, while NEGR1 showed a higher expression level in the obese subjects. No changes were detected for MTCH2, GNPDA2 and MC4R. The expression of KCTD15 was negatively correlated with Body mass index (BMI) and abdominal circumference. The siRNA-mediated knockdown of KCTD15 significantly suppressed cellular lipid accumulation during adipocyte differentiation.

Conclusion: This is the first study to identify the expression patterns of eight obesity-related genes which is connected with adiposity in WAT of overweight people in China. Our study demonstrates that the expression of KCTD15 is associated with obesity in humans. Meanwhile, KCTD15 may play a critical role in the regulation of adipogenesis.

\section{Clinical Trial Registration Number: ChiCTR-RNRC-11001441}

\section{Background}

Obesity is a result of excessive amount of triglycerides storage in adipose tissue, which is the result from a significantly higher amount of calories acquired than that being consumed by human body. White adipose tissue (WAT) is accepted as essential for the regulation energy homeostasis ${ }^{(1)}$, and the aberrantly increased mass of WAT is a phenotype of obesity that is determined by adipocyte number (adipocyte differentiation) and size (fat storage) ${ }^{(2)}$. Studies on the expression profiles of obesity-related genes in adipose tissue have provided valuable information for understanding the development and regulation of obesity ${ }^{(3)}$. Genome-wide association studies (GWAS) has presented a solid proof that candidate obesity-related genes influence the fat level and obesity risk in various populations. An updated map of the human obesity gene published by Rankinen et al, which has become a significant instrument for researchers ${ }^{(4)}$. In addition, A meta-analysis of 15 genome-wide association studies further identified regularly-used obesity-related variables at eight gene lociinclude Fat Mass and Obesity-associated protein(FTO), melanocortin 4 receptor (MC4R), transmembraneprotein18 (TMEM18), potassium channel 
tetramerization domain containing 15 (KCTD15), glucosamine-6-phosphate isomerase 2 (GNPDA2), SH2Badapterprotein1 (SH2B1), mitochondrial carrier homolog $2(\mathrm{MTCH} 2)$ and neuronal growth regulator 1 (NEGR1) ${ }^{(5)}$,and among which the earliest gene loci that was found to contribute to non-syndromic overweight in human is $\mathrm{FTO}{ }^{(6)}$. $\mathrm{SH} 2 \mathrm{~B} 1$ and MC4R are proved to be associated with the regulation of body weight through knockout experiments ${ }^{(7,8)}$. TMEM18 and NEGR1 are likely to have a link with appetite and energy balance via its central sites of action or an influence on adipogenesis in the periphery ${ }^{(9)}$. KCTD15 seems to function as an energy balance regulator ${ }^{(10)}$. GNPDA2 is associated with hexosamine signaling pathway, causing HFD (high fat diet)-fed rats to exhibit the characteristics of obesity and insulin-resistant phenotype. SH2B1 promotes adipocyte differentiation. Plenty of studies have reported that these genes are expressed in adipose tissue on HFD fed or fasted mice, diet-induced obese rats and Zucker diabetic fatty (ZDF) rats ${ }^{(10-13)}$. However, in-depth research is considered necessary to delineate the underlying process for these genes to regulate energy homeostasis and obesity.

\section{Method}

An investigation was conducted to find out how these eight-candidate obesity-related genes are expressed in WAT of normal weight and obese subjects, and the correlation between the expression of the genes and BMI to identify that the genes were significantly associated with obesity. Interestingly, we found that KCTD15 showed the strongest correlation with BMI. KCTD15 was the first identified in human as a BTB domain-containing protein with a conserved C-terminus ${ }^{(14)}$, but the function remains unelucidated. Here, we used the mouse-derived 3T3-L1 pre-adipocyte cell line as an in vitro adipocyte differentiation model to study the function of KCTD15 during adipocyte differentiation ${ }^{(15)}$. The role of KCTD15 in adipogenesis was confirmed by examining its expression profile during 3T3-L1 differentiation. Additionally, the importance of KCTD15 during adipocyte differentiation and lipid accumulation was also further determined by using siRNA-mediated knockdown approaches.

\section{Subject tissue samples}

Based on the fundamental propositions laid out in the Declaration of Helsinki, we were granted permission from the adult subjects or guardians on behalf of the minors participants involved in our study by written before sample collection. Adipose tissue specimens were obtained from 6 overweight subjects, 17 obese subjects ( 14 females, 9 males) and 16 normal weight subjects ( 13 females, 3 males) (aged from 11 to 79 years, with a median of 45 years) who had an elective surgery at Xinqiao Hospital of Army Medical University, Chongqing, China. Demographic data of the studied subjects is shown in Supplementary Table S1, S2. All subjects, who were neither smoker nor under lipid lowering medications, went through thorough clinical phenotyping in the fasted state (12 h, overnight). To calculate the BMI (weight/height squared) $\left(\mathrm{kg} / \mathrm{m}^{2}\right)$, the subject's weight was measured. Besides, the subjects' height was determined using a stadiometer $(\mathrm{cm})$. On the other hand, the abdominal circumference was ascertained utilizing a measuring tape. In order to figure out how prevalent overweight and obesity was, a calculation was performed with a combination of the body mass index (BMI) (normal weight $18.5-23.9 \mathrm{~kg} / \mathrm{m}^{2}$; 
overweight $24-27.9 \mathrm{~kg} / \mathrm{m}^{2}$; obesity $\geq 28 \mathrm{~kg} / \mathrm{m}^{2}$ ) and the explanation of abdominal circumference (abdominal obesity: male $\geq 90 \mathrm{~cm}$, female $\geq 85 \mathrm{~cm}$ ) provided by Working Group on Obesity in China (16). The subjects with cancer, HIV, collagen diseases and syphilis were excluded from the study. We received permission from the studied participants. The project was approved by the local ethics committee of Army Medical University. Before extraction of total RNA and protein, sampling of latest adipose tissue which was conducted intraoperatively and immediately for freezing in liquid nitrogen and stored at -80 ${ }^{\circ} \mathrm{C}$.

\section{RNA extraction and RT-PCR}

Human WAT with TRIzol® Reagent (Invitrogen) was involved for the extraction of total RNA and one microgram of total RNA was applied to synthesize cDNA by using the Revert Aid First Strand cDNA Synthesis Kit (Fermentas, K1611) in line with the requirements specified by the manufacturer, which was followed by PCR amplification with specific forward and reverse primers for 35 to 45 cycles. To enable internal control, GAPDH was utilized. The specific primers were used for the human genes are listed (Supplementary Table S4). The mouse primers of KCTD15 used in the study are as follows:

forward, 5'-ATGGGGAGATTTTCCGCTAC,

reverse, 5'-GGGGCTCCTGCTTTATCC.

A separation of the PCR products was conducted in $3 \%$ agarose gels for a subsequent visualization under UV light. Then, a quantitative analysis was performed to determine the density of the band using Quantity One software (Bio-Rad). The relative expression of all the genes detected were normalized to the GAPDH or $\beta$-actin.

\section{Cell growing under controlled conditions culture and induction of differentiated adipocyte}

While sustaining 3T3-L1 pre-adipocytes, the standard protocol was applied to facilitate them to differentiate $\left.{ }^{(17}\right)$. Briefly, Previous to confluence, Dulbecco's Modified Eagle Medium (DMEM) supplemented with 10\% newborn calf serum (SH30401.01, Hyclone) and 1\% antibiotics

(Penicillin/Streptomycin/Amphotericin B) was involved to grow 3T3-L1 pre-adipocytes (we named the cell confluence day as Day-2, D-2). With 48 hours past cell confluence (Day 0, D0), the cells were induced to differentiation by exposing to differentiation medium containing $1 \mu \mathrm{mol} / \mathrm{L}$ dexamethasone (D2915, Sigma), $0.5 \mathrm{mmol} / \mathrm{L}$ isobutyl methylxanthine (I5879, Sigma), $2 \mu \mathrm{mol} / \mathrm{L}$ rosiglitazone (R2408,Sigma), 10 $\mu \mathrm{g} / \mathrm{mL}$ insulin (16634, Sigma), and 10\% fetal bovine serum (FBS; A15-151, PAA) for three days. When it approached the end of the $3^{\text {rd }}$ day, DMEM supplemented only with $10 \mu \mathrm{g} / \mathrm{mL}$ insulin and $10 \% \mathrm{FBS}$ was used as a substitute of the original substance to grow the cells, and was subjected to replenishment for every two days. Upon the completion of the process to differentiate, a minimum of $90 \%$ of the cells accumulated lipid droplets at Day 10 , for which they were selected as mature adipocytes. 
The siRNA transfection of 3T3-L1 pre-adipocytes was performed as indicated ${ }^{(18)}$. GenePharma was applied to the design and synthesis of mouse KCTD15 siRNA (Shanghai, China). The siRNA sequences are indicated here:

Mouse KCTD15 siRNA \#1 5'-GACGUCUCAAAACUGCUGCUU,

\#2 5'-CAACUGCUGUCCGGAUAAA,

\#3 5'-GGCUACUGUAGACUCAACU;

Negative control siRNA, 5'-UUCUCCGAACGUGUCACGU.

3T3-L1 preadipocytes were grown to $30-50 \%$ confluency in $35 \mathrm{~mm}$ dishes and transfected with siRNA oligonucleotides by applying Lipofectamine ${ }^{\mathrm{TM}}$ RNAiMAX (Invitrogen, 13778-150) according to the instructions of the manufacturer. Forty-eight hours later, the knockdown efficiency was detected by RTPCR to facilitate differentiation, the standard differentiation protocol was applied.

\section{Oil Red 0 dyeing}

Subsequent to obtaining the mature adipocytes by in vitro differentiation, the lipid droplets in the mature adipocyte cells were monitored by adopting the approach of modified Oil Red $O$ staining ${ }^{(19)}$. In brief, the cells were washed twice with PBS before fixed with 10\% formaldehyde for a duration of $1 \mathrm{~h}$ at room temperature. Afterwards, the cells were washed twice with $60 \%$ isopropanol and then dyed by applying Oil Red 0 solution for $1 \mathrm{~h}$. Images of the Oil Red O-stained cells were derived from using with Leica DMI3000 B inverted microscope.

\section{Triglyceride assay}

Triglycerides can be quantified with the triglyceride assay kit E1003-2 (Applygen Technologies Inc, Beijing, China). Cells ended up being harvested by trypsinization, rinsed three times with PBS and then lysed in lysis buffer. The supernatant was assayed in line with the requirements laid out by the manufacturer.

\section{Western blotting}

Total protein lysate was extracted from 3T3-L1 cells at different time points during differentiation with RIPA buffer (20 mM Tris-HCl, pH7.4, 20\% glycerol, 0.5\% NP40, 1 mM MgCl2, 150 mM NaCl, 1 mM EDTA, 1 mM EGTA.). Protein concentrations were measured using the Bradford Assay, and $100 \mu \mathrm{g}$ protein extracts were subjected to SDS-PAGE. Then proteins were transferred to a PVDF membrane, blocked with $5 \%$ nonfat milk and incubated with first antibodies (anti-KCTD15, Abcam ab106373, 1: 1000; anti- $\beta$-Tubulin, Sigma t-5293, 1: 10000) for $1 \mathrm{~h}$ at R.T. After incubation with secondary antibody against mouse (Dakocytomation, p0161, 1:10,000) or rabbit (Dakocytomation, p0448, 1:10,000) for $1 \mathrm{~h}$ at R.T, the membrane was visualized by ECL Western Blotting Detection Kit (RNP2232, GE healthcare).

\section{Statistical Analysis}


All statistics were interpreted as the mean \pm SEM. The correlation coefficient, Student's t-test (for single comparison) and one-way ANOVA (for group comparisons) were involved to analyze data where appropriate using SPSS 16.0 for Windows (SPSS, part of IBM Corporation; Somers, NY). Tukey's post hoc test was applied under the circumstance of a necessary comparison among individual groups. $P<0.05$ was regarded as being of statistical importance.

\section{Results}

\section{Clinical characteristics of the study subjects}

Body mass index (BMI) is a prevalent indicator to assess individuals as normal weight or obese. In China, obesity is defined as an individual with BMI of $28 \mathrm{~kg} / \mathrm{m}^{2}$ and above, and which has been used as a guideline on preventing people from getting obese or overweight ${ }^{(20)}$. To date, eight genes have already been widely accepted as associated with obesity according to the genome-wide association studies. To further explore the relationship between these obesity-related genes and obesity, adipose tissue specimens from 23 obese subjects and 16 normal weight subjects were collected. Compared with normal subjects, who had an average BMI of $21.06 \pm 1.79$ and abdominal circumference of $74.13 \pm 4.47$, The obese subjects had a significantly higher average BMI of $28.78 \pm 1.78$ and abdominal circumference of $105.6 \pm 6.07$.

\section{The expression of eight obesity-related genes in human adipose tissue and the correlation of these genes with BMI}

To detect the RNA expression level of eight obesity-related genes, total RNA was isolated from adipose tissues of both normal weight and obese subjects. RNA was later amplified by RT-PCR, and the representative PCR results are shown in Fig.1A. PCR products were quantified with Quantity One software (BioRad) and the relative expression of each gene was normalized to GAPDH. As indicated by Fig.1B, the expression of KCTD15 and SH2B1 in obese individuals were substantially significant relative to the individuals with normal weight, whereas the expression of TMEM18 and FTO was slightly less significant in the obese individuals than with normal people. In comparison, the expression of NEGR1 increased significantly among those obese subjects $(P=0.0092)$. Nonetheless, there was no notable variation in gene expressions of the MTCH2, GNPDA2 and MC4R between two groups.

An investigation was carried out into how the eight genes are expressed in both standard weight and obese individuals and determined whether these genes are linked to obesity by analyzing the correlation coefficient for gene expression and BMI. Those variables with $P$ values less than 0.05 were considered to be correlated. Thus, TMEM18, SH2B1, FTO and KCTD15 were negatively correlated with BMI, while NEGR1 was positively correlated with BMI (Fig.1C). The representative correlation curve for KCTD15 showed the most significant negative correlation with BMI (Fig.1D) and correlation with abdominal circumference (Supplement TableS3). 
We further explored the role of KCTD15 during adipogenesis by using 3T3-L1 pre-adipocytes in vitro differentiation system. Firstly, the standard induction protocol detailed in the method part was applied to facilitate 3T3-L1 cells to differentiate, and cells were collected at Day-2, Day 0, Day 2, Day5 and Day10. Sequentially, the expression of KCTD15 at different time points were spotted by PCR with specific PCR primers and the density of the PCR products were quantified with Quantity One software. As shown in Fig.2A, KCTD15 expression was high at the beginning of differentiation. Meanwhile, the KCTD15 level throughout the differentiation process was determined and normalized to $\beta$-actin (Fig.2B). The results showed that KCTD15 expression increased at day-0 before decreasing and reaching the lowest level in mature adipocytes (day10) (Fig.2C, 2D).

\section{KCTD15 deficiency compromised adipocyte differentiation and lipid accumulation in 3T3-L1 cells}

For further investigated how KCTD15 worked in adipogenesis, the knockdown strategy was applied. Firstly, KCTD15 specific siRNA was transiently transfected into 3T3-L1 pre-adipocytes, while non-targeting siRNA was used as negative controls. As shown in Fig.3A, the expression of KCTD15 decreased obviously after siRNA transfection, suggesting that the knock down efficiency of KCTD15 was high (Fig.3A). Then the cells were induced to differentiation and ten days after the induction, the extent of adipocyte differentiation was checked by Oil Red $O$ dyeing. Intriguingly, the differentiation of KCTD deficient cell was significantly inhibited compared to the negative control (Fig.3B). In addition, another indicator of adipogenesis, lipid accumulation, was also measured in both control and KCTD15 knockdown cells through measuring the triglyceride level in the cells. Consistent with the impact made by KCTD15 knockdown on adipocyte differentiation, the level of accumulated triglycerides was also lower in the KCTD15 deficient cells than the control cells (Fig.3C). These measurements were taken in triplicate and had a $P$ value of 0.0220 . Taken together, these data suggest that KCTD15 performs a crucial part for adipogenesis.

\section{Discussion}

Studies on the association between the obesity-related genes identified by GWAS and obesity suggested the need of further research into the biological function of these genes, which promoted us to discover how the genes are expressed in normal weight and obese subjects. Therefore, as far as the existing research is concerned, the focus is on the variation of mRNA level among these candidate obesity-related genes in adipose tissue from normal weight and obese subjects. Among these genes, SH2B1 had been reported to promote adipocyte differentiation through the control of PPARY levels ${ }^{(21)}$. NEGR1 acted as the focal point of transcript network in relation to obesity ${ }^{(22)}$. FTO mediated adipogenesis and adipocyte lipid content by regulating gene expression ${ }^{23}$. TMEM18 and NEGR1 had been implicated in obesity by regulating the central nervous system (CNS) ${ }^{(11)}$. KCTD15 was shown to inhibit neural crest formation by regulate transcription factor AP-2 ${ }^{(24)}$. No obvious changes of $\mathrm{MTCH} 2$, GNPDA2 and MC4R were detected. Intriguingly, the expression of $\mathrm{MTCH} 2$, which is abundant in human WAT, was shown to be higher in obese women ${ }^{(25)}$. Meanwhile, it was found that TMEM18 and FTO expression were lower in obese 
subjects. The expression of SH2B1 and KCTD15 were significantly decreased in obese subjects, whereas NEGR1 was increased in this group. Interestingly, it was also illustrated that the expression of KCTD15 was down-regulated in the adipose tissue of obese rats ${ }^{(10)}$. We are the first to confirm that the expression of KCTD15 is less obvious among obese people but with a notable negative association with BMI displayed, which is used to identify the degree of obesity. Studies have suggested that KCTD15 is capable of regulation of various biological processes in relation to obesity, such as glucose metabolism and fat metabolism ${ }^{(5,19)}$. However, the difference made by KCTD15 in adipogenesis is still unknown. Therefore, we focused on the role of KCTD15 in adipogenesis.

Aberrant and excessive fat accumulation was defined as obesity. Fat accumulation was determined by adipocyte number (adipocyte differentiation) and size (fat storage). Similarly, 3T3-L1 pre-adipocyte differentiation is composed of the growth inhibition of proliferating pre-adipocytes (Day 0 ), coordinated reentry into the cell cycle with clonal expansion (Days 1-2), postmitotic growth inhibition (Days 3-4), associated terminal differentiation and lipid accumulation (Days 4-10) (23). Among these differentiation stages, the clonal expansion stage played a necessary and indispensable role in differentiation. With this research, it was revealed that KCTD15 was expressed at a high level during initial stages (pre-adipocytes, Days 0-2) and then became lower in the later stages (mature adipocytes, Days 5-10). The data indicated that KCTD15 may be essential for the early mitotic events. Indeed, we revealed that KCTD15 knockdown in 3T3-L1 leads to the inhibition of adipocyte differentiation and lipid accumulation.

Our in vitro adipocyte differentiation study showed that KCTD15 was crucial for adipocyte differentiation initiation, whereas, in comparison, there was a downgrade in the regulation of how KCTD15 was expressed in adipose tissues obtained from overweight human subjects when compared to those obtained from normal-weight controls. This seemingly paradoxical data might be due to the fact that white adipose tissue in human terminally differentiated. Consequently, it was reasonable to observed that the expression of KCTD15 in adipose tissue from overweight subjects was low, because of our in vitro experiments clearly demonstrated that KCTD15 expression was down-regulated in the terminal stage of adipocyte differentiation.

Notably, the sequential biphasic changes in KCTD15 gene expression during adipogenesis were not unique. During adipocyte differentiation, the protein level of KCTD15 increased in the early stage and then markedly decreased in the later stage. It was also shown that knockdown of KCTD15 blocked adipocyte differentiation. It had been verified that KCTD15 could regulate the expression of transcription factor AP-2 (24). During adipocyte differentiation, transcription of genes that generated the adipocyte phenotype could be activated by CCAAT enhancer-binding protein- $a$ (C/EBPa) coordinately ${ }^{(25-26)}$. Before mitotic clonal expansion occurs, C/EBPa gene ended up being silenced because of the repression of AP-2 ${ }^{(24)}$.We hypothesized that KCTD15 inhibited transcription factor AP-2 expression and eventually affected adipocyte differentiation via suppression of C/EBPa. However, more evidence is required to determine the underlying mechanism whereby KCTD15 regulates adipocyte differentiation. 
In summary, we elucidated the expression patterns of eight obesity-associated genes in adipose tissue and obese subjects. It was discovered that KCTD15 expression level was closely related to BMI. In addition, our in vitro adipocyte differentiation study demonstrated that KCTD15 had a potential to perform a critical part at the initial stages of adipocyte differentiation. Nevertheless, in-depth researches are required to figure out the mechanism contributing to adipocyte differentiation by KCTD15.

\section{Conclusion}

Our study suggested that KCTD15 could regulate the adipogenesis and the expression of KCTD15 was associated with obesity in humans. Therefore, KCTD15 could play a critical part in the regulation of adipogenesis and obesity.

\section{Declarations}

\section{Ethical Aspects}

Permission for this research was granted by the Human Research Ethics of Xinqiao Hospital of Army Military Medical University, under the number of ChiCTR-RNRC-11001441, and all patients signed the informed consent form.

\section{Ethics approval and consent to participate}

The clinical trial registration number is ChiCTR-RNRC-11001441, and we were granted permission from the adult subjects.

\section{Consent for publication}

All authors agree to publish.

\section{Availability of data and materials}

The data that support the findings of this study are available

\section{Competing interests}

The authors have no conflicts of interest to disclose.

\section{Funding}

This study was supported by a Clinical Research Award from Army Medical University and a Grant-in-Aid from the Disease Genomics and Individualized Medicine Laboratory, Beijing Institute of Genomics, Chinese Academy of Sciences. 


\section{Author's contributions}

JX was responsible for the collection and preservation of clinical specimen, conduct of all the experiments and data collection and analysis; JLD was responsible for manuscript modification and online submission $\triangle \mathrm{YD}$ was responsible for chart modification; $\mathrm{MW}$ was responsible for communication with patients and collection of clinical specimens as required in patient's informed consent; KBZ was responsible for analyzing statistics and modifying manuscript. ZHX was responsible for subject's design and statistical analysis and modification of manuscript and online submission.

\section{Acknowledgements}

We appreciated XZ for RNA and protein manipulation, adipocyte differentiation experiments, $\mathrm{YY}$ for assisting adipocyte differentiation experiments, CL, BH (Department of Orthopedic, Xinqiao Hospital, Army Medical University) for assisting in the collection of clinical specimens, QCY (Division of Molecular Cardiology, Department of Medicine, College of Medicine, Texas A\&M Health Science Center) for reading and modifying the manuscript.

\section{References}

1. Stern JH, Rutkowski JM, Scherer PE. Adiponectin, Leptin, and Fatty Acids in the Maintenance of Metabolic Homeostasis through Adipose Tissue Crosstalk. Cell Metab. 2016; 23:770-84.

2. Choe SS, Huh JY, Hwang IJ, Kim JI, Kim JB. Adipose Tissue Remodeling: Its Role in Energy Metabolism and Metabolic Disorders. Front Endocrinol. 2016; 7:30.

3. Magkos F, Fraterrigo G, Yoshino J, et.al. Effects of Moderate and Subsequent Progressive Weight Loss on Metabolic Function and Adipose Tissue Biology in Humans with Obesity. Cell Metab. 2016; 23:591-601.

4. Locke AE, Kahali B, Berndt SI, et.al. Genetic studies of body mass index yield new insights for obesity biology. Nature. 2015; 518:197-206.

5. Felix JF, Bradfield JP, Monnereau C, et.al. Genome-wide association analysis identifies three new susceptibility loci for childhood body mass index. Hum Mol Genet. 2016; 25:389-403.

6. Loos RJ, Yeo GS. The bigger picture of FTO: the first GWAS-identified obesity gene. Nat Rev Endocrinol. 2014; 10:51-61.

7. Giuranna J, Volckmar AL, Heinen A, et.al. The Effect of SH2B1 Variants on Expression of Leptin- and Insulin-Induced Pathways in Murine Hypothalamus. Obes Facts. 2018; 11:93-108.

8. Mosialou I, Shikhel S, Liu JM, et.al. MC4R-dependent suppression of appetite by bone-derived lipocalin 2. Nature. 2017; 543:385-390.

9. Speakman JR. Functional analysis of seven genes linked to body mass index and adiposity by genome-wide association studies: a review. Hum Hered. 2013; 75:57-79.

10. Gutierrez-Aguilar R, Kim DH, Woods SC, Seeley RJ. Expression of new loci associated with obesity in diet-induced obese rats: from genetics to physiology. Obesity. 2012; 20:306-12. 
11. Ronkainen J, Huusko TJ, Soininen R, al. Fat mass- and obesity-associated gene Fto affects the dietary response in mouse white adipose tissue. Sci Rep. 2015; 5:9233.

12. Yoganathan $P$, Karunakaran $S$, Ho MM, Clee SM. Nutritional regulation of genome-wide association obesity genes in a tissue-dependent manner. Nutrition \& metabolism. 2012; 9:65

13. Schmid PM, Heid I, Buechler C,al. Expression of fourteen novel obesity-related genes in Zucker diabetic fatty rats. Cardiovasc Diabetol. 2012; 11:48.

14. Hotta K, Nakamura M, Nakamura T, et.al. Association between obesity and polymorphisms in SEC16B, TMEM18, GNPDA2, BDNF, FAIM2 and MC4R in a Japanese population. J Hum Genet. 2009; 54:727-31.

15. MacDougald OA, Lane MD. Transcriptional regulation of gene expression during adipocyte differentiation. Annu Rev Biochem. 1995; 64:345-373.

16. Gao P, Shen J. An empirical analysis on the determinants of overweight and obesity in china. Applied Economics. 2017; 49:1923-1936.

17. Zhu HJ, Pan $H$, Zhang XZ, et.al. The effect of myostatin on proliferation and lipid accumulation in 3t3-I1 preadipocytes. Journal of molecular endocrinology. 2015; 54:217-26.

18. Li X, Kim JW, Gronborg M, Urlaub H, Lane MD, Tang QQ. Role of cdk2 in the sequential phosphorylation/activation of c/ebpbeta during adipocyte differentiation. Proceedings of the National Academy of Sciences of the United States of America. 2007; 104:11597-11602.

19. Zebisch K, Voigt V, Wabitsch M, Brandsch M. Protocol for effective differentiation of 3t3-I1 cells to adipocytes. Analytical biochemistry. 2012; 425:88-90.

20. Jiang $Y, X u Y, B i$ YF, et.al. Prevalence and trends in overweight and obesity among Chinese adults in 2004-10: Data from three nationwide surveys in china. The Lancet. 2015; 386: S77.

21. Rui L. Sh2b1 regulation of energy balance, body weight, and glucose metabolism. World journal of diabetes. 2014; 5:511-526.

22. Boender AJ, van Gestel MA, Garner KM, Luijendijk MC, Adan RA. The obesity-associated gene negr1 regulates aspects of energy balance in rat hypothalamic areas. Physiological reports. 2014; 2.

23. Martin Carli JF, LeDuc CA, Zhang Y, Stratigopoulos G, Leibel RL. Fto mediates cell-autonomous effects on adipogenesis and adipocyte lipid content by regulating gene expression via 6ma DNA modifications. Journal of lipid research. 2018; 59:1446-1460.

24. Zarelli VE, Dawid IB. Inhibition of neural crest formation by kctd15 involves regulation of transcription factor ap-2. Proceedings of the National Academy of Sciences of the United States of America. 2013; 110:2870-5.

25. Linhart HG, Ishimura-Oka K, DeMayo F, et.al. C/ebpalpha is required for differentiation of white, but not brown, adipose tissue. Proceedings of the National Academy of Sciences of the United States of America. 2001; 98:12532-12537.

26. Jiang MS, Lane MD. Sequential repression and activation of the ccaat enhancer-binding proteinalpha (c/ebpalpha) gene during adipogenesis. Proceedings of the National Academy of Sciences of 


\section{Figures}

$A$

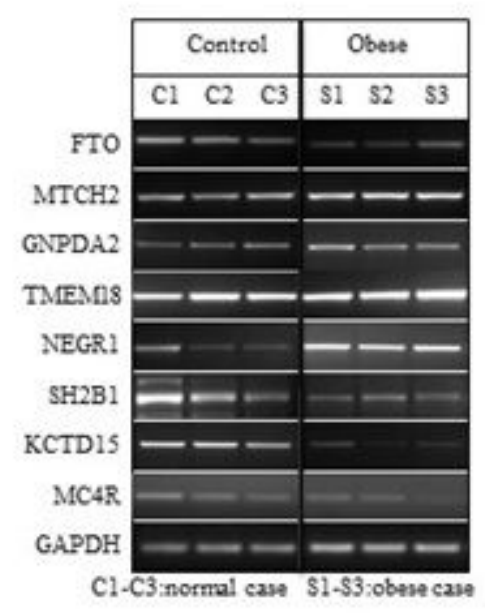

c

\begin{tabular}{|c|c|c|}
\hline Gene Name & Pvalve & $\begin{array}{c}\text { Pearson } \\
\text { correlation }\end{array}$ \\
\hline FTO & 0.509 & -0.109 \\
\hline MTCH2 & 0.954 & 0.010 \\
\hline GNPDA2 & 0.598 & 0.090 \\
\hline TMEM18 & $0.020 *$ & -0.375 \\
\hline NEGR1 & $0.011 *$ & 0.423 \\
\hline SH2B1 & $0.001 * *$ & -0.0524 \\
\hline KCTD15 & $0.004 * *$ & -0.0469 \\
\hline MC4R & 0.918 & 0.017 \\
\hline
\end{tabular}

B

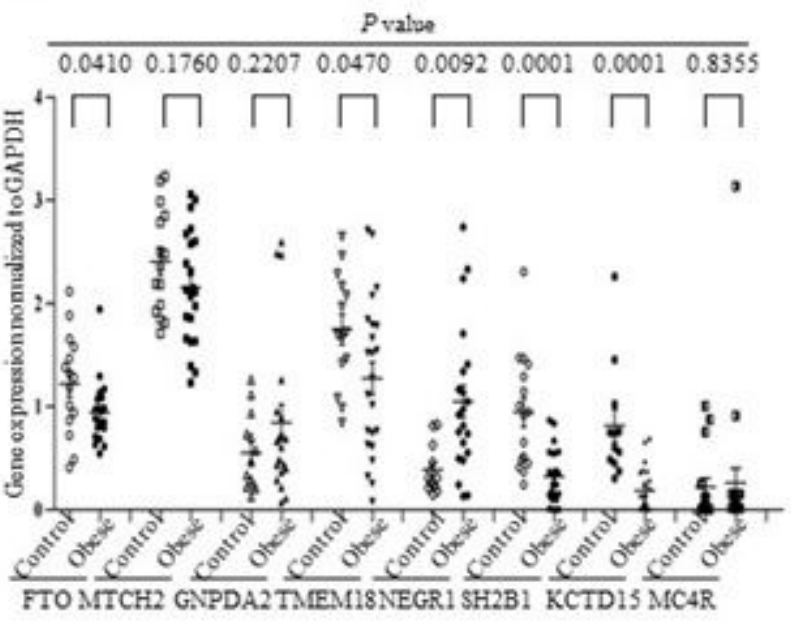

D

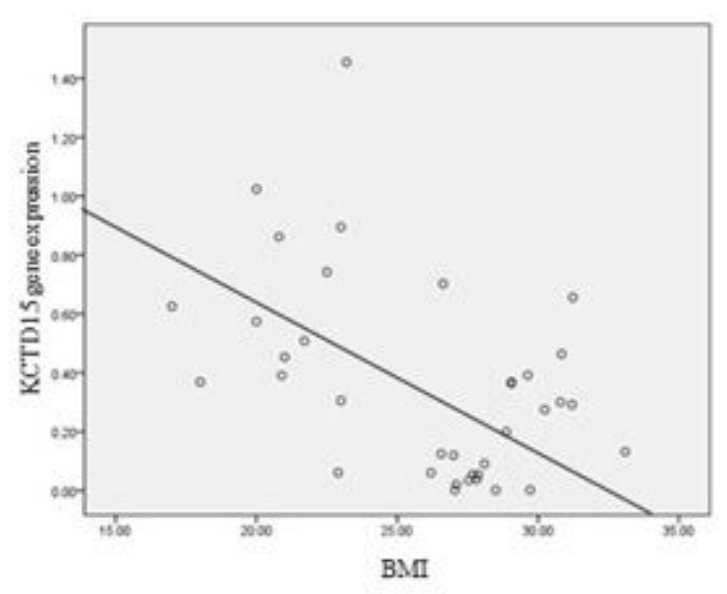

\section{Figure 1}

The mRNA expressions of eight obesity-related genes in the adipose tissue from normal and obese subjects and correlation with obesity.(A) Total RNAs from adipose tissues of 16 normal subjects and 23 obese subjects were isolated for mRNA expression measurement. Representative PCR results of the eight genes were presented. GAPDH was used as a control. (B) All PCR products were quantified by Quantity One software and normalized to GAPDH levels. The relative expressions of eight genes in all subjects were presented as closed or open geometric figures. The values in the figure are shown as mean \pm SEM. $P$ $<0.05$ was considered statistically significant. (C) The correlation coefficient between expressions of genes and BMI was analyzed with SPSS 16.0 software. * and ** presents Pearson correlation $<0.05$ and $<0.01$ respectively, indicating that the values are significantly correlated. The person value less than 0 means negative correlation while more than 0 means positive correlation. A Pearson correlation coefficient close to +1 or -1 indicates that the two variables are highly correlated (positively or 
negatively, respectively). A correlation coefficient between 0 and +0.30 or between 0 and -0.30 was considered of having no correlation between the two variables. (D) The expression of KCTD15 was negatively-correlated with BMI. $\mathrm{P}$ and $\mathrm{r}$ values are given for each simple regression plot.

A

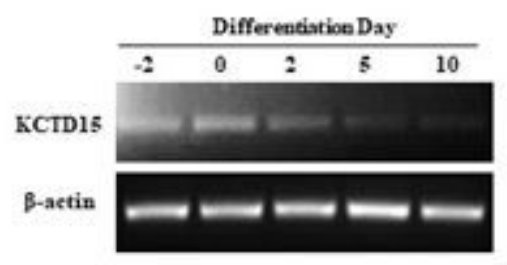

C

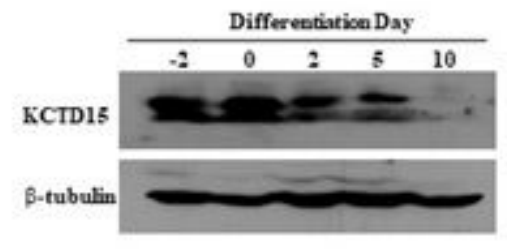

D

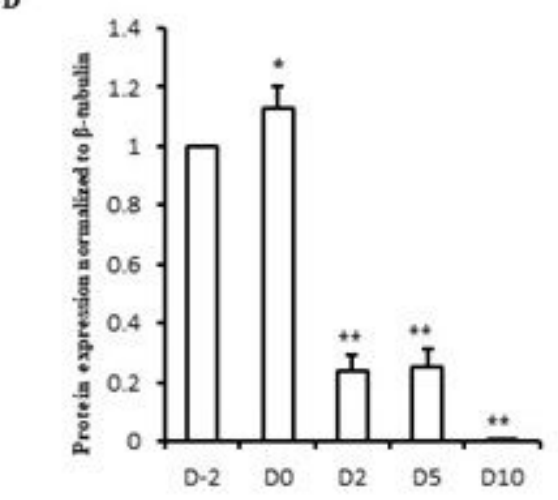

Figure 2

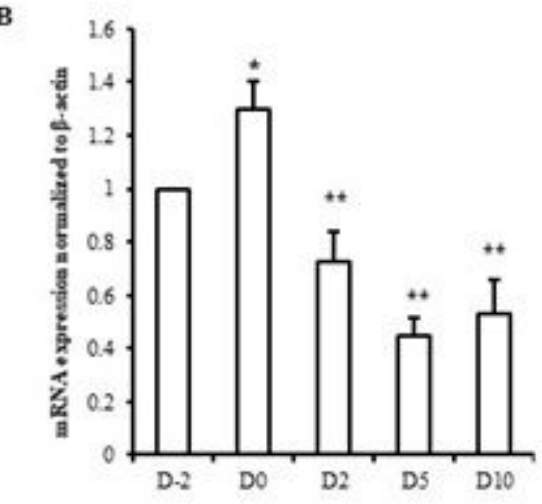

The expression of KCTD15 was decreased during adipocyte differentiation. (A) Differentiation of 3T3-L1 preadipocytes (Day-2) were induced by hormone cocktail. Total RNAs were isolated for quantification of KCTD15 by RT-PCR at different time points (Day-2/0/2/5/10). $\beta$-actin was used as an internal control. (B) PCR products were quantified by Quantity One software. The values were presented as density of PCR products normalized to $\beta$-actin levels. (C) Cell lysates were collected at different stages of differentiation (Day-2/0/2/5/10) with RIPA buffer, and subjected to western blot detection. KCTD15 protein was detected with specific antibody. $\beta$-tubulin was used as a loading control. (D) Group data showing the protein expressions of KCTD15 normalized to $\beta$-tubuli expression. ${ }^{*} P<0.05$ versus the stage of differentiation Day-2, \# P $<0.05$, \#\# $P<0.01$ versus the stage of differentiation Day-0. 
A

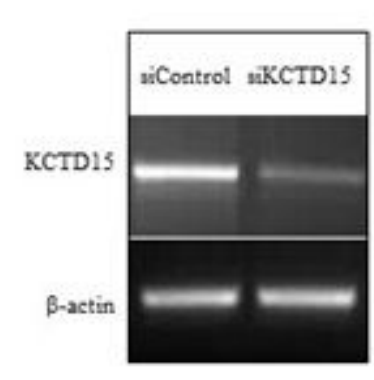

c

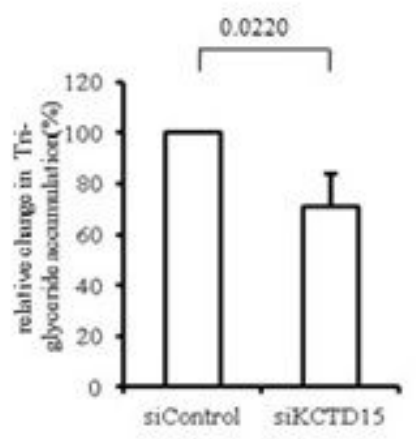

B

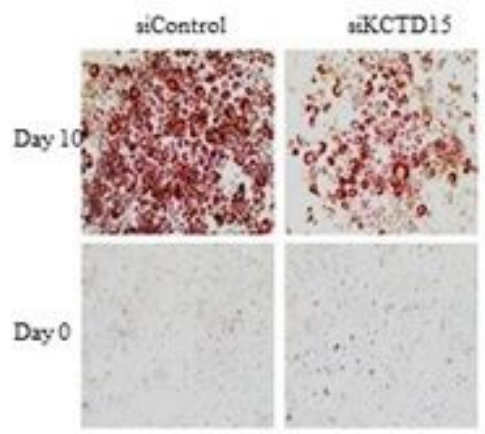

\section{Figure 3}

Deficiency of KCTD15 inhibits adipocyte differentiation and lipid accumulation. (A) Preadipocyte 3T3-L1 cells were transfected with negative control or KCTD15 specific siRNA, respectively, and subjected to induction of differentiation. Knockdown efficiency was tested by semi-quantity RT-PCR after 48 h. $\beta$-actin was used as an internal control. (B) Oil Red 0 staining of cells was performed at Day-0 and Day 10 in both negative control and KCTD15 specific siRNA treated cells. (C) The triglyceride content was measured with a triglyceride kit as mg triglycerides/mg protein. Relative content represents the percentage of the knockdown group normalized by the control group. Data are presented as means \pm SEM of 3 independent experiments performed in triplicate. * Statistical significance $(P<0.05)$.

\section{Supplementary Files}

This is a list of supplementary files associated with this preprint. Click to download.

- SupplementaryTables.pdf 